\title{
Two-Sided Altruism in an OLG Model with Incomplete Markets and Default*
}

\author{
Wilfredo L. Maldonado** \\ Jaime Orrillo ${ }^{* * *}$
}

\begin{abstract}
This paper shows the existence of equilibrium and its non-triviality (i.e. there exist, in all periods, both trade and default in the security markets) in an overlapping generation model with incomplete markets. This result is obtained by assuming that agents in the economy exhibit two-sided altruism in the following way: each agent may leave a financial inheritance to his only offspring (forward altruism) in the last period of his life, and in the same period, the descendant may accumulate debts in the name of the agent i.e., the father (backward altruism). Thus, if the descendant does not honor the commitment acquired in the name of his antecessor, he will suffer penalties in his utility function. To avoid the excessive issuance of debt, we shall suppose that the agent (the father) suffers a disutility in proportion to the net value of the debt left to his descendant (the son).
\end{abstract}

Keywords: Overlapping Generations, Default, Altruism, Incomplete Markets.

JEL Codes: D52, D64.

${ }^{*}$ Submitted in October 2005. Revised in September 2006.

Wilfredo L. Maldonado acknowledges CNPq for financial support 300059/99-0(RE). Jaime Orrillo also acknowledges CNPq for financial support 301218/2004-8.

${ }^{* *}$ Catholic University of Brasilia, Brazil. SGAN 916, Módulo B, 70790-160 Asa - Norte, Brasília - DF. E-mail: wilfredo@pos.ucb.br

${ }^{* * *}$ Catholic University of Brasilia, Brazil

Brazilian Review of Econometrics

v. $26, \mathrm{n}^{\circ} 2$, pp. 291-308 Nov 2006 


\section{Introduction}

Currently, almost all economic agents (from a single consumer to a government) go to the financial markets to request loans. They do this either to finance individual consumption (in the case of the former) or to finance some social or economic project or even the public debt in the case of the latter. At the same time, great amounts of default have also been registered in the world economy; and this is because some individuals or governments do not honor their commitments. Therefore, creditors must take this phenomenon into account whenever they decide to lend (or invest). Usually they (international bankers) anticipate a possible default on the part of the countries due to political or financial crises. This leads creditors to suspend (at least temporarily) credits until confidence is recovered. To see how the fear of a default on the part of a government causes financial crises we refer to Cole and Kehoe (2000).

Since the pioneering work of Dubey et al. (1990), where default was incorporated in a context of general equilibrium, many have been the works (mainly on the existence of equilibrium) regarding this subject. For a more recent version of the paper above, see Dubey et al. (2005). Once the default becomes part of the strategies of a borrower, lenders should not expect to receive the whole of their returns (due to the adverse selection); nor should borrowers plan on selling great quantities of an asset for the fear of default penalties. Although many legal penalties are rarely used to force agents to meet their commitments, authors like Zame (1993), and Araújo et al. (1996), Araújo et al. (1998) followed Dubey et al. (1990) with respect to the use of default penalties. In these models, the penalties were assessed directly in terms of utility. However, these have been strongly criticized because of their subjective nature. This opened the possibility of seeking new mechanisms to enforce commitments which should not take into account any individual characteristics.

Geanakoplos and Zame $(1995,2002)$ required debtors to buy durable goods whenever they wanted to sell some assets available for trading. Since then, the advantages of collaterals have been explored by several authors. For instance, Orrillo (2001) used them to dispense the non-negative of ex-post income in a continuum setting; Araújo et al. (2002) and Orrillo (2002) to avoid Ponzi-Schemes; and Kubler and Schmedders (2003) to facilitate the computing of equilibrium. In this study we will adopt utility penalties, not collaterals, for defaulting.

In this paper we model two-sided altruism via financial markets in an OLG economy with incomplete markets, where the agents live two periods and the father and son coexist during one period of time. This type of generation structure is not typical in OLG models (except that of Laitner (1993)), where the new generation appears in the period following the death of the antecessor.

Two-sided altruism is modeled in the following manner: each agent may leave financial inheritance to his only offspring in his last period of life (forward altruism), and in the same period, the descendant may accumulate debts in the name 
of that agent i.e., the father (backward altruism). In technical terms this may be viewed as "inheritance of the financial debt" left by the father to the son. The provision of this credit is made using the same financial instruments that the son uses to finance his own consumption (credit cards, payment obligations, etc.). In this way, any default on this debt must be penalized on the son's utility since the son is responsible for the debt issued in his father's favor. Lastly, to avoid the excessive issuance of debt, we shall suppose that the agent (the father) suffers a disutility in proportion to the net value of the debt left to his descendant (the son). To simplify our analysis we shall suppose that the agents may default only on debts issued in favor of their antecessors.

Overlapping generation models had their origins in the works of Allais (1947) and Samuelson (1958). They were conceived to deal with monetary features. For a survey about this theme the reader may consult Geanakoplos (1987). OLG models with incomplete markets without default were analyzed by Cass et al. (1992), and Gottardi (1996). Although these works include incomplete markets, they also consider money, which is not our specific concern in this paper. Recently, Florenzano et al. (2001) have extended OLG models for the case in which uncertainty is modeled by a continuum of states. This paper does not attempt to extend any OLG model mentioned here. Rather, it provides a framework for a better understanding of how credits are transferred from parents to offspring and how these can assume the responsibility of the debt issued in favor of the parents.

Our first goal is to demonstrate that default is consistent with the orderly function of markets in the OLG setting given above. This is obtained under the usual assumptions regarding utilities, initial endowments and the financial structure. Our other main goal is to prove the existence of a non-trivial competitive equilibrium. That is, there exist, in all periods, both trade and default in the securities markets. The non-triviality of the equilibrium is guaranteed by assuming the existence of: a) an individual who, although possessing society's initial endowment, would be willing to default on debt issued in his antecessor's name whenever he wanted to increase his private consumption during old age and b) the individual's antecessor, who, although also possessing society's initial endowment, would not be willing to bequeath (net) financial inheritances to his successor, whenever he wanted to increase his private consumption during old age.

For the existence argument we adopt the methodology of truncated economies. We show that the sequence of truncated economies, obtained by cutting the horizon of time as well as the domain of decision variables, has an equilibrium. This result is derived from Debreu's approach applied to the generalized game associated with each truncated economy. The existence result then follows from asymptotic properties of truncated equilibria. Finally, we prove the non-triviality of equilibrium using our hypothesis above, which is related to the eventual selfish behavior of some agents.

The contribution of this paper can be viewed from two perspectives: First, 
unlike early results about the existence of equilibrium with incomplete markets, ours explicitly shows the existence of trading in the financial markets. This can be useful when the researcher is seeking to choose a model which represents real facts with effective financial trading. Second, the fact of having a strictly positive default rate serves as a theoretical foundation to explain the incorporation of the credit risk in the interest rates of some assets.

The paper is organized as follows: In Section 2, we describe the model of our OLG economy. In Section 3, we outline the model's hypotheses, state our main result and prove the non-triviality of equilibrium. In Section 4, we offer some concluding remarks. Lastly, we provide an Appendix containing the proof of existence of the equilibrium.

\section{The Model}

We consider an OLG model where agents live for two periods. The markets are incomplete and agents may default. The time is discrete and is indexed by integers. The stochastic structure of the model is given by an infinite event tree without any initial node and a finite number $S$ of branches at each node of the tree. It is assumed that each agent, in the first period of his life, coexists with his antecessor and with his successor in the terminal period of his life. Both have access to the financial markets where $J$ real assets are traded.

Our analysis is specialized for the case in which each young individual is the single heir of an older individual. To formalize this we introduce the offspring function $f: V_{n} \rightarrow J_{n}$ which associates to each agent $h$ (which is old in period $n$ ) his descendant $f(h)$ (which is young in period $n$ ). In a similar way $f^{-1}(h)$ will denote the antecessor of the young individual $h$.

\section{Fundamentals of the economy}

There is only one infinitely divisible good for trading. The set of histories $S^{n}$ is defined to be the $S^{Z_{n}}$ consisting of all truncated sequences of states of nature. Here $Z_{n}=\{\ldots, n\}$. A typical element of $S^{n}$ is denoted by $\tilde{s}_{n}$, and in most cases, we will delete the lower index from $\tilde{s}_{n}$ if the period of time $n$ is understood. Lastly, we denote $\tilde{s}=\left(\tilde{s}^{-}, s\right)$, being $\tilde{s}^{-} \in S^{n-1}$ and $s \in S$.

\section{Financial structure}

Each asset $j$ is characterized by a sequence of promises $\left\{A_{n}^{j}(\tilde{s})\right\}_{n \in Z}$ where $A_{n}^{j}(\tilde{s}) \in$ $R_{+}$is the payoff ${ }^{1}$ that the holder of one share of assets $j$ receives at node $\tilde{s} \in S^{n}$; by a couple of sequences of penalties for defaulting and for leaving net debts $\left\{\lambda^{j}(\tilde{s})\right\},\left\{\gamma^{j}(\tilde{s})\right\}$ respectively; and limits on short sales $v \in R_{+}^{J}$. Securities and the only available commodity are traded in spot markets. It is assumed that $J<S$ in each period, i.e., markets are incomplete.

${ }^{1}$ They are in terms of the consumption good. 
Agents

Endowments and preferences of the agents are assumed to be affected by uncertainty, but their births and deaths are not. The common consumption and portfolio sets are $C_{n}=L^{o}\left(S^{n}, R_{+} \times R_{+}^{S}\right)$, and $K_{n}=L^{o}\left(S^{n}, R_{+}^{J} \times R_{+}^{J S}\right) \times L^{o}\left(S^{n}, R_{+}^{J} \times\right.$ $\left.R_{+}^{J S}\right)$, respectively. Here, $L^{o}\left(S^{n}, R_{+}^{k}\right)$ represents all $\mathbb{R}_{+}^{k}\left(\right.$ deve_ser $\left.: R_{+}^{k}\right)$ - functions defined on the set of all partial histories $S^{n}$. Thus, the agent $h \in \mathcal{H}$ who is young in period $n$ is characterized by his utility function $u^{h}: R^{S+1} \rightarrow R$, defined for each $c \in C_{n}$ and each history $\tilde{s} \in S^{n}$ by $u^{h}(c(\tilde{s}))$, and his initial endowment $\omega^{h} \in L^{o}\left(S^{n}, R_{+} \times R_{+}^{S}\right)$.

An economy is defined as a vector

$$
\mathcal{E}=\left[\left(u^{h}, \omega^{h}\right)_{h \in \mathcal{H}},\left(A^{j}, \lambda^{j}, \gamma^{j}, v_{j}\right)_{j \in J}\right]
$$

Endogenous variables

These variables consist of macro variables and individual choice variables. The macro variables are asset prices $q=\left\{q_{n}\right\}_{n \in Z}$ with $q_{n}: S^{n} \rightarrow R_{+}^{J}$, and default rates $k_{n}=\left\{k_{n}\right\}_{n \in Z}$ with $k_{n}: S^{n} \rightarrow[0,1]^{J}$. The individual choice variables are consumption $\left\{\mathbf{c}_{n}^{h}\right\}_{n \in Z}$, with $\mathbf{c}_{n}^{h}(\tilde{s})=\left(c_{o}^{h}(\tilde{s}),\left(c_{s}^{h}(\tilde{s})\right)_{s \in S}\right) \in R_{+} \times R_{+}^{S}, \forall \tilde{s} \in S^{n}$; purchasing and selling of assets $\left\{\mathbf{y}_{n}^{h}, \mathbf{z}_{n}^{h}\right\}_{n \in Z}$, with $\mathbf{y}_{n}^{h}(\tilde{s})=\left(y_{o}^{h}(\tilde{s}),\left(y_{s}^{h}(\tilde{s})\right)_{s \in S}\right) \in$ $R_{+}^{J} \times R_{+}^{J S}$, and $\mathbf{z}_{n}^{h}(\tilde{s})=\left(z_{o}^{h}(\tilde{s}),\left(z_{s}^{h}(\tilde{s})\right)_{s \in S}\right) \in R_{+}^{J} \times R_{+}^{J S}, \forall \tilde{s} \in S^{n}$, and lastly deliveries $D_{n}^{h j}(\tilde{s})=\left(D_{n+1, s}^{h j}(\tilde{s})\right)_{s \in S}$ with $D_{n+1, s}^{h j}(\tilde{s}) \in\left[0, A_{n+1}^{j}(\tilde{s}, s) z_{j}^{f^{-1}(h)}(\tilde{s})\right]$. Lastly, define $\mathbf{q}_{n}(\tilde{s})=\left(q_{n}(\tilde{s}), q_{n+1}(\tilde{s},).\right)$.

The budget set and payoff

For each history $\tilde{s} \in S^{n}$, the budget set of agent $h$, who lives in periods $[n, n+1]$, is defined as follows. Let $\mathbf{q}_{n}(\tilde{s})$ be the asset prices and $k_{n+1}(\tilde{s},$.$) the default rate. If$ $y^{f^{-1}(h)}(\tilde{s})$ is the financial inheritance left by $h^{\prime}$ s parent and $z^{f^{-1}(h)}(\tilde{s})$ is the debt issue decision of $h^{\prime}$ s parent that has to be authorized and assumed by $h$, then $h^{\prime} \mathrm{s}$ budget set is the set consisting of consumption plans, portfolio plans and delivery plans $x=\left(c_{0},\left(c_{s}\right), y_{0},\left(y_{s}\right), z_{0},\left(z_{s}\right),\left(D_{s}\right)\right) \in R^{(S+1)+2 J(S+1)+J S}$, which satisfies the following budget constraints: for all $\tilde{s} \in S^{n}$,

$$
\begin{gathered}
c_{o}+q_{n}(\tilde{s}) y_{o} \leq \omega_{n}^{h}(\tilde{s})+q_{n}(\tilde{s}) z_{o} \\
c_{s^{\prime}}+q_{n+1}\left(\tilde{s}, s^{\prime}\right) y_{s^{\prime}}+\sum_{j \in J} D_{s^{\prime}}^{j}+\sum_{j \in J} A_{n+1}^{j}\left(\tilde{s}, s^{\prime}\right) z_{o j} \leq \omega_{n+1}^{h}\left(\tilde{s}, s^{\prime}\right)+q_{n+1}\left(\tilde{s}, s^{\prime}\right) z_{s^{\prime}} \\
+\sum_{j \in J}\left(1-k_{n+1}^{j}\left(\tilde{s}, s^{\prime}\right)\right) A_{n+1}^{j}\left(\tilde{s}, s^{\prime}\right)\left\{y_{o j}+y_{j}^{f^{-1}(h)}(\tilde{s})\right\}, \forall s^{\prime} \in S
\end{gathered}
$$

Budget constraint (1) is standard in the literature. Budget constraint (2) deserves an explanation. Reported on the right side of the inequality are the total 
wealth of individual $h$ in his old age. The first term $\omega_{n+1}^{h}$ is the initial endowment, the second $z_{s^{\prime}}$ is an indebtedness decision of agent $h$ when old, which must be issued by $f(h)$ in the favor of the father $h$. The value of this loan $q_{n+1}\left(\tilde{s}, s^{\prime}\right) z_{s^{\prime}}$ is granted by $f(h)$ to $h$ and thus it appears as wealth in the second period of $h^{\prime}$ s life. The third term $\sum_{j \in J}\left(1-k_{n+1}^{j}\left(\tilde{s}, s^{\prime}\right)\right) A_{n+1}^{j}\left(\tilde{s}, s^{\prime}\right)\left\{y_{o j}+y_{j}^{f^{-1}(h)}(\tilde{s})\right\}$ is the total return of investments (discounted by the default rate of the economy) that agent $h$ made when young plus the financial inheritance left by $f^{-1}(h)$. On the left side of the inequality (2) are the expenditures of $h$ when old. These are consumption $c_{s}$, investments $q_{n+1}\left(\tilde{s}, s^{\prime}\right) y_{s^{\prime}}$ which will be left as inheritance to $f(h)$, total financial payments $\sum_{j \in J} A_{n+1}^{j}\left(\tilde{s}, s^{\prime}\right) z_{o j}$ of debt acquired when young and the (partial) payment $\sum_{j \in J} D_{s^{\prime}}^{j}$ of the debt acquired in the name of his father. Remember that for the sake of simplicity we are assuming that the agent honors his own financial commitments issued when young.

In addition, we will assume uniform bounds on short sales. That is,

$$
\exists v \in R_{++}^{J(S+1)}: \mathbf{z} \leq v
$$

We can then define, for each history $\tilde{s} \in S^{n}$, the budget set for $h \in J_{n}$ as the set

$$
B_{n}^{h}\left(\mathbf{q}_{\mathbf{n}}, k_{n+1}, y^{f^{-1}(h)}, z^{f^{-1}(h)}\right)
$$

consisting of all the $x:=(\mathbf{c}(\tilde{s}), \mathbf{y}(\tilde{s}), \mathbf{z}(\tilde{s}), D(\tilde{s})) \in R_{+}^{(S+1)+2 J(S+1)+J S}$ such that (1), (2) and (3) are satisfied, and where $k_{n+1}=\left(k_{n+1}^{j}\right)_{j \in J}$.

The following functional form will be assumed for the payoff function:

$$
\begin{aligned}
U^{h}(\mathbf{c}, \mathbf{y}, \mathbf{z}, D, \tilde{s}) & =u^{h}(\mathbf{c}(\tilde{s}))-\sum_{j \in J} \sum_{s^{\prime} \in S} \gamma^{j}\left(\tilde{s}, s^{\prime}\right) q_{n+1}^{j}\left(\tilde{s}, s^{\prime}\right)\left(z_{s^{\prime} j}(\tilde{s})-y_{s^{\prime} j}(\tilde{s})\right) \\
& -\sum_{j \in J} \sum_{s^{\prime} \in S} \lambda^{j}\left(\tilde{s}, s^{\prime}\right)\left[A_{n+1}^{j}\left(\tilde{s}, s^{\prime}\right) z_{j}^{f^{-1}(h)}(\tilde{s})-D_{s^{\prime}}^{j}(\tilde{s})\right]^{+}
\end{aligned}
$$

The first term corresponds to the utility of the private consumption of agent $h$ in the two periods of his life. The second one is the disutility suffered by leaving net debts to his son $f(h)$. In fact, the term

$$
\sum_{j \in J} \sum_{s^{\prime} \in S} \gamma^{j}\left(\tilde{s}, s^{\prime}\right) q_{n+1}^{j}\left(\tilde{s}, s^{\prime}\right) z_{s^{\prime} j}(\tilde{s})
$$

is a disutility suffered by $h$ resulting from the loan that his son $f(h)$ acquires in his favor. This avoids large amounts of debt issued in the economy. On the other hand, the term 


$$
\sum_{j \in J} \sum_{s^{\prime} \in S} \gamma^{j}\left(\tilde{s}, s^{\prime}\right) q_{n+1}^{j}\left(\tilde{s}, s^{\prime}\right) y_{s^{\prime} j}(\tilde{s})
$$

represents an additional utility of $h$ because of leaving financial inheritances (using financial assets) to his offspring. In this way we can interpret the term

$$
\sum_{j \in J} \sum_{s^{\prime} \in S} \gamma^{j}\left(\tilde{s}, s^{\prime}\right) q_{n+1}^{j}\left(\tilde{s}, s^{\prime}\right)\left(z_{s^{\prime} j}(\tilde{s})-y_{s^{\prime} j}(\tilde{s})\right)
$$

as a disutility of $h$ resulting from the net debt left to $f(h)$. This is a kind of forward altruism which uses financial instruments and does not directly incorporate consumption which is classic in the literature. Finally, the last term

$$
\sum_{j \in J} \sum_{s^{\prime} \in S} \lambda^{j}\left(\tilde{s}, s^{\prime}\right)\left[A_{n+1}^{j}\left(\tilde{s}, s^{\prime}\right) z_{j}^{f^{-1}(h)}(\tilde{s})-D_{s^{\prime}}^{j}(\tilde{s})\right]^{+}
$$

represents the $h$ 's disutility of not paying the debts made when young in the favor of his father. This term is standard in models with penalties in the utility with the difference that the loan acquired by $h$ was decided by his antecessor and it was to finance the consumption of his father when he was old. The simple feature of $h$ issuing debt in the name of his father already incorporates backward altruism in the model. Thus, our structure describes the two-sided altruism which can be modeled via financial instruments.

\section{Equilibrium}

We are now ready to define what equilibrium means.

Definition 1 An equilibrium for $\mathcal{E}$ is a sequence of commodity and asset prices and default rates $\left(\mathbf{q}_{n}, k_{n}\right)_{n \in Z}$, and an allocation ${ }^{2}\left(\mathbf{c}_{n}^{h}, \mathbf{y}_{n}^{h}, \mathbf{z}_{n}^{h}, D^{h}\right)_{h \in \mathcal{H}}$ such that

1. (Optimality) For each period $n$ and history $\tilde{s} \in S^{n}$, $x_{n}^{h}(\tilde{s}):=\left(\mathbf{c}_{n}^{h}(\tilde{s}), \mathbf{y}_{n}^{h}(\tilde{s}), \mathbf{z}_{n}^{h}(\tilde{s}), D^{h}(\tilde{s})\right)$ maximizes $U^{h}(\mathbf{c}, \mathbf{y}, \mathbf{z}, D, \tilde{s})$ subject to his budget set $B^{h}\left(\mathbf{q}_{\mathbf{n}}, k_{n+1}, y^{f^{-1}(h)}, z^{f^{-1}(h)}\right)$;

2. (Markets Clear) Both commodity and asset markets clear at each period $n \in Z$, i.e., $\forall \tilde{s} \in S^{n}$ we have $\sum_{h \in J_{n}}\left[c_{o}^{h}(\tilde{s})+c^{f^{-1}(h)}(\tilde{s})\right]=\sum_{h \in J_{n}}\left[\omega_{o}^{h}(\tilde{s})+\right.$ $\left.\omega^{f^{-1}(h)}(\tilde{s})\right]$, and $\sum_{h \in J_{n}}\left[y_{o}^{h}(\tilde{s})+y^{f^{-1}(h)}(\tilde{s})\right]=\sum_{h \in J_{n}}\left[z_{o}^{h}(\tilde{s})+z^{f^{-1}(h)}(\tilde{s})\right]$;

3. (Correct Anticipation of the Payment Rate) For every $n \in Z$ and history $\tilde{s} \in$ $S^{n}$ given, the payment rate $t_{n+1}^{j}\left(\tilde{s}, s^{\prime}\right):=\left(1-k_{n+1}^{j}\left(\tilde{s}, s^{\prime}\right)\right)$ for the next period, for each state $s^{\prime} \in S$, will be equal to what is paid $\sum_{h \in J_{n}}\left[A_{n+1}^{j}\left(\tilde{s}, s^{\prime}\right) z_{o j}^{h}(\tilde{s})+\right.$

${ }^{2}$ The variable $D$ is not in boldface because each agent $h \in J_{n}$ will only default when old. Thus, the sub-index $n+1$ will be understood. 
$\left.D_{s^{\prime}}^{h j}(\tilde{s})\right]$ divided by the total debt in the economy $A_{n+1}^{j}\left(\tilde{s}, s^{\prime}\right) \sum_{h \in J_{n}}\left[z_{o j}^{h}(\tilde{s})+\right.$ $\left.z_{j}^{f^{-1}(h)}(\tilde{s})\right], j \in J$ provided that $\sum_{h \in J_{n}}\left[z_{o j}^{h}(\tilde{s})+z_{j}^{f^{-1}(h)}(\tilde{s})\right] \neq 0$.

\section{Assumptions and Main Results}

Our results are established under the following assumptions:

H1) $\forall h \in \mathcal{H}, u^{h}: R_{+} \times R_{+}^{S} \rightarrow R$ is a continuous, strictly increasing and concave function;

H2) For all $h \in \mathcal{H}: w^{h}>>0$ and $\sup _{h \in \mathcal{H}}\left\|w^{h}\right\|<\infty$ and

H3) For all asset $j$ and for all $\tilde{s} \in S^{n}, \sup _{n} A_{n}^{j}(\tilde{s})<\infty$.

The previous assumptions are standard to guarantee existence in infinitehorizon economies, so they do not need any commentary.

Theorem 1 (Existence) Under H1-H3 the OLG economy $\mathcal{E}$ always has an equilibrium.

To prove this theorem one uses the methodology of truncated economies together with Debreu's method (see Debreu (1952)). Although our model considers a single good, the result of existence can be extended straightforwardly in the case where there is more than one good, and where the assets are real. ${ }^{3}$ For that reason, we have provided, in the appendix, a proof when $L \geq 1$ and the assets are real.

\subsection{Non-trivial equilibrium}

Next, we precisely define what a non-trivial equilibrium means.

Definition 2 (Non-trivial equilibrium)

An equilibrium is said to be non-trivial if for each period $n$, there exists an asset $j_{o}$ and a history $\left(\tilde{s}_{o}, s_{o}^{\prime}\right) \in S^{n} \times S$ such that $\sum_{h \in V_{n+1}}\left[z_{s_{o}^{\prime} j_{o}}^{h}\left(\tilde{s}_{o}\right)+z_{o j_{o}}^{f(h)}\left(\tilde{s}_{o}, s_{o}^{\prime}\right)\right] \neq 0$, and there exist $h \in J_{n}$ such that, $0 \leq D_{n+1}^{h j_{o}}\left(\tilde{s}_{o}, s_{o}^{\prime}\right)<A_{n+1}^{j}\left(\tilde{s}_{o}, s_{o}^{\prime}\right) z_{j}^{f^{-1}(h)}\left(\tilde{s}_{o}\right)$.

This definition points out that for an equilibrium to be non-trivial there must be, in all periods, trading in the financial markets at least for one asset $j_{o}$ and one state $s_{o}^{\prime}$, and someone is defaulting. In Araújo et al. (1998) another definition for non-triviality is assumed, namely, that there must be trade or everybody is honoring his financial commitments. ${ }^{4}$

\footnotetext{
${ }^{3}$ Those that pay in terms of bundles of goods.

${ }^{4}$ We found our definition more appropriate. For example, in a pure exchange economy, non-triviality of the equilibrium means that there is exchange indeed. In an economy with 
The following assumptions concerning separability and an individual's selfishness guarantees the non-triviality of the equilibrium

H4) The utility function $u^{h}: R_{+}^{S+1} \rightarrow R$ is separable in the states, and differentiable. That is, $u^{h}(c)=\sum_{s=o}^{S} \bar{u}_{s}^{h}\left(c_{s}\right)$, with $\bar{u}_{s}^{h}: R_{+} \rightarrow R$ strictly increasing and strictly concave;

H5) For each $n, \exists h_{o} \in J_{n}, \exists\left(\tilde{s}_{o}, s_{o}^{\prime}\right) \in S^{n+1}: \forall j$,

$$
\begin{aligned}
\left(\bar{u}_{s_{o}^{\prime}}^{h_{o}}\right)^{\prime}\left(\omega\left(\tilde{s}_{o}, s_{o}^{\prime}\right)\right)>\lambda^{j}\left(\tilde{s}_{o}, s_{o}^{\prime}\right) \text { and }\left(u_{s_{o}}^{f^{-1}\left(h_{o}\right)}\right)^{\prime}\left(\omega\left(\tilde{s}_{o}\right)\right)>\gamma^{j}\left(\tilde{s}_{o}\right) \text { where } \\
\omega\left(\tilde{s}_{o}, s_{o}^{\prime}\right)=\sum_{h \in V_{n+1}}\left[\omega_{n+1}^{h}\left(\tilde{s}_{o}, s_{o}^{\prime}\right)+\omega_{n+1}^{f(h)}\left(\tilde{s}_{o}, s_{o}^{\prime}\right)\right] ; \omega\left(\tilde{s}_{o}\right) \\
=\sum_{h \in V_{n}}\left[\omega_{n}^{h}\left(\tilde{s}_{o}\right)+\omega_{n}^{f(h)}\left(\tilde{s}_{o}\right)\right]
\end{aligned}
$$

Assumption H5 affirms that in each period there is a state of the nature with at least one selfish agent with a selfish antecedent, in the sense that the agent values his consumption in old age more than the payment of the debt issued in his father's favor. Similarly, the agent's antecessor also values his consumption in old age more than the financial bequest he left.

From Theorem 1 it follows that each young agent is maximizing his payoff subject to his budget set. Therefore, there exist Lagrange's multipliers $\alpha_{n}^{h}(\tilde{s})=$ $\left(\alpha_{o}^{h}(\tilde{s}),\left(\alpha_{s^{\prime}}^{h}(\tilde{s})\right)_{s^{\prime} \in S}\right) \in R_{+}^{S+1}$ such that Kuhn-Tucker conditions are satisfied.

Lemma 1 The following inequalities are true for each history $\tilde{s} \in S^{n}$, for any state of nature $s^{\prime} \in S$ to be revealed in the period $n+1$, and for any agent $h \in J_{n}$.

1. $0 \leq \lambda^{j}\left(\tilde{s}, s^{\prime}\right)\left\{\left[A_{n+1}^{j}\left(\tilde{s}, s^{\prime}\right) z_{j}^{f^{-1}(h)}(\tilde{s})-D_{s}^{j}\right]^{+}-\left[A_{n+1}^{j}\left(\tilde{s}, s^{\prime}\right) z_{j}^{f^{-1}(h)}(\tilde{s})-D_{s^{\prime}}^{h j}(\tilde{s})\right]^{+}\right\}$ $-\alpha_{s^{\prime}}^{h}(\tilde{s})\left(D_{s^{\prime}}^{h j}(\tilde{s})-D_{s^{\prime}}^{j}\right)$;

2. $0 \leq\left(\gamma^{j}\left(\tilde{s}_{n}, s^{\prime}\right)-\alpha_{s^{\prime}}^{h}(\tilde{s})\right) q_{n+1, j}\left(\tilde{s}, s^{\prime}\right)\left(z_{s^{\prime} j}-z_{s^{\prime}}^{h}(\tilde{s})\right)$;

3. $u^{h}\left(\left(c_{o}^{h}(\tilde{s}),\left(c_{s^{\prime}}\right)_{s^{\prime} \in S}\right)-u^{h}\left(\mathbf{c}_{n}^{h}(\tilde{s})\right) \leq \alpha_{s^{\prime}}^{h}(\tilde{s})\left(c_{s^{\prime}}-c_{s^{\prime}}^{h}(\tilde{s})\right)\right.$.

Proof After substituting into the Kuhn-Tucker conditions (obtained from the young individual's payoff maximization problem) all the variables by their optimal values except $D_{s^{\prime}}$, Item 1 follows; except $z_{j s^{\prime}}$, Item 2 follows; and except

production, non-triviality would mean the existence of production (not only trading). In a financial economy, non-triviality would mean the existence of trade. Therefore, in a financial economy with possibility of default, non-triviality must mean existence of trade and default at the same time. 
$c_{s^{\prime}}^{h}(\tilde{s})$, Item 3 follows.

Item 1 implies that $\forall\left(\tilde{s}, s^{\prime}\right) \in S^{n+1}$.

$D_{s^{\prime}}^{h j}(\tilde{s})=A_{n+1}^{j}\left(\tilde{s}, s^{\prime}\right) z_{j}^{f^{-1}(h)}(\tilde{s}) \Rightarrow \alpha_{s^{\prime}}^{h}(\tilde{s}) \leq \lambda^{j}\left(\tilde{s}, s^{\prime}\right)$ provided $z^{f^{-1}(h)}(\tilde{s})>0$

Item 2 implies that $\forall\left(\tilde{s}, s^{\prime}\right)$, and $\forall j$ with $q_{n+1, j}\left(\tilde{s}, s^{\prime}\right)>0$ :

$$
z_{s^{\prime} j}^{h}(\tilde{s})=0 \Rightarrow \alpha_{s^{\prime}}^{h}(\tilde{s}) \leq \gamma^{j}\left(\tilde{s}, s^{\prime}\right)
$$

Lastly, Item 3 implies that, provided that $u^{h}$ is differentiable, $\forall\left(\tilde{s}, s^{\prime}\right)$, the following inequality is held

$$
\left(\bar{u}_{s^{\prime}}^{h}\right)^{\prime}\left(\mathbf{c}_{n}^{h}(\tilde{s})\right) \leq \alpha_{s^{\prime}}^{h}(\tilde{s})
$$

Theorem 2 (Non-triviality) If H1-H5 are satisfied, then the equilibrium is nontrivial.

Proof H4 and (6) imply that

$$
\forall h \in J_{n},\left(\bar{u}_{s^{\prime}}^{h}\right)^{\prime}\left(\omega_{s l}\left(\tilde{s}, s^{\prime}\right)\right)<\alpha_{s^{\prime}}^{h}(\tilde{s})
$$

Putting $h=h_{o}$, and $\left(\tilde{s}, s^{\prime}\right)=\left(\tilde{s}_{o}, s_{o}^{\prime}\right)$ satisfying H5 one has both $\alpha_{s_{o}^{\prime}}^{h_{o}}\left(\tilde{s}_{o}\right)>$ $\lambda^{j}\left(\tilde{s}_{o}, s_{o}^{\prime}\right)$ and $\alpha_{s_{o}^{\prime}}^{f^{-1}\left(h_{o}\right)}\left(\tilde{s}_{o}^{-}\right)>\gamma^{j}\left(\tilde{s}_{o}^{-}, s_{o}^{\prime}\right)$ for all $j$. Since $q_{n}\left(\tilde{s}_{o}\right) \in \triangle^{J-1}, \exists j_{o}$ : $q_{n, j_{o}}\left(\tilde{s}_{o}\right) \neq 0$. Using (5) one has $z_{j_{o}}^{f^{-1}\left(h_{o}\right)}\left(\tilde{s}_{o}\right)>0$ which implies trading in period $n$. Thus, the young $h_{o} \in J_{n}$ will have to pay a strictly positive debt (made in favor of his antecedent) $A_{n+1}^{j}\left(\tilde{s}_{o}, s^{\prime}\right) z_{j_{o}}^{f^{-1}\left(h_{o}\right)}\left(\tilde{s}_{o}\right)>0$.

Using (4), the agent $h_{o}$ will pay $D_{s_{o}^{\prime}}^{h_{o} j_{o}}\left(\tilde{s}_{o}\right)<A_{n+1}^{j_{o}}\left(\tilde{s}_{o}, s_{o}^{\prime}\right) z_{j_{o}}^{f^{-1}\left(h_{o}\right)}\left(\tilde{s}_{o}\right)$ since $\alpha_{s_{o}^{\prime}}^{h_{o}}\left(\tilde{s}_{o}\right)>\lambda^{j}\left(\tilde{s}_{o}, s_{o}^{\prime}\right)$. Thus, there is default.

An immediate implication of the non-triviality of the equilibrium is that there exists trading for the asset $j_{o}$ so that its payment rate is well defined and is strictly less than one because there is no full delivery for the asset $j_{o}$.

\section{Summary and Concluding Remarks}

In this paper, we model an OLG economy with incomplete markets and the possibility of default. In this setting, agents use the financial instruments available to exercise altruism from parents to offspring and conversely. In the OLG classic models altruism is modeled by incorporating the son's utility of consumption into the father's utility of consumption or vice versa. In our model backward altruism is modeled through the debt acquisition in favor of the father by his descendant. 
Of course, the agent's default on this debt will result in utility penalties which are common to these situations. On the other hand, forward altruism is shown through two channels. The first one is through the satisfaction felt by the father in leaving a net positive inheritance of financial credits to his offspring. The second one is through regret ${ }^{5}$ (disutility) felt by the father in leaving financial debts that the son will assume in the name of his father. The net value of these two operations provokes forward altruism.

It is useful to note that both manners of modeling the exercise of altruism are completely feasible in practice. That is, the agents use the same financial instruments to finance their own consumption and that of the antecedent. They also bequeath financial assets as inheritance to their offspring.

Under the usual hypotheses regarding utility functions, initial endowments and asset returns, equilibrium in our economy always exists. Lastly, in order to guarantee the non-triviality of the equilibrium, it was sufficient to assume, in each period, the existence of two individuals: the antecessor and his descendant, where the former prefers, in some state, to consume rather than to bequeath financial bequests, and the latter prefers, in the same state, to consume rather than to pay the financial debt issued in the name of antecessor.

Finally, it is important to note that our model can not be seen as a particular case of an infinite horizon economy model since altruistic behavior is modeled as a financial bequest rather than the embodiment of the consumption utility of the agent's descendant inside himself. This seems to be more adequate in economies with financial markets.

\section{References}

Allais, M. (1947). Économie et Intérêt. Imprimerie Nationale, Paris.

Araújo, A., Monteiro, P., \& Páscoa, M. R. (1996). Infinite horizon incomplete markets with a continuum os states. Mathematical Finance, 6(2):119-132.

Araújo, A., Monteiro, P., \& Páscoa, M. R. (1998). Incomplete markets. continuum of states and default. Economic Theory, 11:205-213.

Araújo, A., Páscoa, M. R., \& Torres, J. P. (2002). Collateral avoids ponzi schemes in incomplete markets. Econometrica, 70(4):1613-1638.

Cass, D., Green, R. C., \& Spear, S. E. (1992). Stationary equilibria with incomplete markets and overlapping generations. International Economic Review, 33:495512.

Cole, H. L. \& Kehoe, T. J. (2000). Self-fulfilling debt crises. Review of Economic Studies, 67(1)(230):91-116.

\footnotetext{
${ }^{5}$ We are assuming that if the father regrets leaving debts to his son it is because he loves his son.
} 
Debreu, G. (1952). A social equilibrium existence theorem. Proceedings of the National Academy of Sciences, 38:886-893.

Dubey, P., Geanakoplos, J., \& Shubik, M. (1990). Default and efficiency in a general equilibrium model with incomplete markets. Cowles Foundation Discussion Paper, 773R.

Dubey, P., J., G., \& Shubik, M. (2005). Default and punishment in general equilibrium. Econometrica, 73(1):1-37.

Florenzano, M., Gourdel, P., \& Páscoa, M. R. (2001). Overlapping generations models with incomplete markets. Journal of Mathematical Economics, $36(3): 201-218$.

Geanakoplos, J. (1987). The overlapping generations model of general equilibrium. In Eatwell, J., Milgate, M., \& Newman, P., editors, The New Palgrave, pages 767-779. MacMillan, London.

Geanakoplos, J. \& Zame, W. (1995). Default, collateral and derivatives. Unpublished manuscript.

Geanakoplos, J. \& Zame, W. (2002). Collateral and the enforcement of intertemporal contracts. Yale University Working Paper.

Gottardi, P. (1996). Stationary monetary equilibria in overlapping generation models with incomplete markets. Journal of Economic Theory, 71:75-89.

Hildenbrant, W. (1974). Core and Equilibria of a Large Economy. Princeton University Press, Princeton.

Kubler, F. \& Schmedders, K. (2003). Stationary equilibria in asset-pricing models with incomplete markets and collateral. Econometrica, 71(6):1767-1793.

Laitner, J. (1993). Long-run equilibria with borrowing constraints and altruism. Journal of Economics Dynamics and Control, 17:65-96.

Orrillo, J. (2001). Default and exogenous collateral in incomplete markets with a continuum of states. Journal of Mathematical Economics, 35:151-165.

Orrillo, J. (2002). Making promises in infinite-horizon economies with default. Catholic University of Brasilia Working Paper.

Samuelson, P. A. (1958). An exact consumption-loan model of interest with or without the social contrivance of money. The Journal of Political Economy, 66:647-480.

Zame, W. (1993). Efficiency and the role of default when security markets are incomplete. American Economic Review, 83:1142-1164. 


\section{Appendix A}

\section{Truncated Economy}

In this section, we are going to consider a sequence of truncated economies $\left\{\mathcal{E}^{N}\right\}_{N \in Z^{+}}$where truncation concerns the horizon and the set where the decision variables are chosen. The proof of the existence of equilibrium will be done considering $L \geq 1$ commodities. Thus, the vector of commodity prices $p_{n}: S^{n} \rightarrow R_{+}^{L}$ will be introduced in the obvious way. Therefore, the budget set of each young agent $h \in J_{n}$ in $\mathcal{E}^{N}$ is, for each history $\tilde{s} \in S^{n}$, the set

$$
B_{n}^{h N}\left(\mathbf{p}_{n}, \mathbf{q}_{n}, k_{n+1}, y^{f^{-1}(h N)}, z^{f^{-1}(h N)}\right)
$$

consisting of all $x_{n}^{N} \in[0, N]^{L(S+1)+2 J(S+1)+J S}$ such that (1), (2) and (3) are satisfied; where $x_{n}^{N}=\left(\mathbf{c}_{n}^{N}(\tilde{s}), \mathbf{y}_{n}^{N}(\tilde{s}), \mathbf{z}_{n}^{N}(\tilde{s}), D^{N}(\tilde{s})\right)$, and $-N \leq n \leq N$. To shorten the notation, the upper index $N$ will be deleted from all choice variables (even from prices, payment and default rates), but one must not forget the dependence of these variables on $N$ when we study their asymptotic properties, i.e., when $N$ goes to infinity. We will assume that $\left(p_{n}, q_{n}\right): S^{n} \rightarrow \triangle^{L-1} \times \triangle^{J-1}, \forall n \in Z$ and in particular for $-N \leq n \leq N$.

For each integer number $N \geq 0$ we define the following generalized game played by the finite number $2 \mathrm{NH}$ of young agents and $l+f$ additional players; where $l=2 N+1$ are auctioneers and the other $f=(2 N+1) J$ players are fictitious agents choosing default rates. Denote this game by $\mathcal{G}_{N}$, which is described as follows: given the history $\tilde{s} \in S^{n}$.

- Each young agent $h \in J_{n}$, with $n=-N+1, \ldots, N-2$, maximizes $U^{h}$ in the constrained strategy set;

$$
B_{n}^{h N}\left(\mathbf{p}_{n}, \mathbf{q}_{n}, k_{n+1}, y^{f^{-1}(h N)}, z^{f^{-1}(h N)}\right)
$$

Notice that the agent $h \in J_{-N}$ will not pay any debts or receive credits since he does not inherit them. Therefore, the deliveries do not appear in his budget constraints, nor in his payoff. Similarly, the young people that live in period $N-1$ will not bequeath any credits or debts because there will be nobody to pay them. So the value of the financial bequest will be missing in both his old budget constraint and payoff.

- The auctioneer of the period $n \in[-N, N]$ chooses $\left(p_{n}\left(\tilde{s}_{n}\right), q_{n}\left(\tilde{s}_{n}\right)\right) \in \triangle^{L-1} \times$ $\triangle^{J-1}$ in order to maximize;

$$
p_{n}\left(\tilde{s}_{n}\right) \sum_{h \in J_{n}}\left[c_{o}^{h}\left(\tilde{s}_{n}\right)+c^{f^{-1}(h)}(\tilde{s})-\omega_{o}^{h}\left(\tilde{s}_{n}\right)-\omega^{f^{-1}(h)}(\tilde{s})\right]
$$




$$
+q_{n}\left(\tilde{s}_{n}\right) \sum_{h \in J_{n}}\left[y_{o}^{h}\left(\tilde{s}_{n}\right)+y^{f^{-1}(h)}(\tilde{s})-z_{o}^{h}\left(\tilde{s}_{n}\right)-z^{f^{-1}(h)}(\tilde{s})\right]
$$

- Each of the remaining $f$ fictitious agents of state $s^{\prime} \in S$ chooses $t_{n+1}^{j}\left(\tilde{s}, s^{\prime}\right)=$ $1-k_{n+1}^{j}\left(\tilde{s}, s^{\prime}\right) \in[0,1]$ in order to minimize

$$
\begin{gathered}
\left\{t_{n+1}^{j}\left(\tilde{s}, s^{\prime}\right) p_{n+1}\left(\tilde{s}, s^{\prime}\right) A_{n+1}^{j}\left(\tilde{s}, s^{\prime}\right) \sum_{h \in J_{n}}\left[y_{o j}^{h}(\tilde{s})+y_{j}^{f^{-1}(h)}\left(\tilde{s}_{n}\right)\right]\right. \\
\left.-\sum_{h \in J_{n}}\left[p_{n+1}\left(\tilde{s}, s^{\prime}\right) A_{n+1}^{j}\left(\tilde{s}, s^{\prime}\right) z_{o j}^{h}(\tilde{s})+D_{s^{\prime}}^{h j}(\tilde{s})\right]^{+}\right\}^{2}
\end{gathered}
$$

Remarks Due to the particularity of the budget sets of young people who live in periods $-N$ and $N-1$ respectively, both the auctioneers' and fictitious agents' payoffs will be different from those of other young people of the economy $\mathcal{E}^{N}$.

For instance, the auctioneer of the period $-N$ chooses commodity and asset prices in that period in order to maximize

$$
p_{-N}\left(\tilde{s}_{-N}\right) \sum_{h \in J_{-N}}\left[c_{o}^{h}\left(\tilde{s}_{-N}\right)-\omega_{o}^{h}\left(\tilde{s}_{-N}\right)\right]+q_{-N}\left(\tilde{s}_{-N}\right) \sum_{h \in J_{-N}}\left[y_{o}^{h}\left(\tilde{s}_{-N}\right)-z_{o}^{h}\left(\tilde{s}_{-N}\right)\right]
$$

The auctioneer of the period $N$ acts in a similar way. On the other hand, the fictitious agent, who chooses the default rate, will only enter from period $-N+2$ on since in that period the old agent will begin to default on the antecessors' promises.

Our next objective is to show that $\mathcal{G}^{N}$ has an equilibrium in pure strategies, since this equilibrium will correspond to an equilibrium of the truncated economy $\mathcal{E}^{N}$.

Lemma 2 Under assumptions H1-H3, the generalized game $\mathcal{G}^{\mathcal{N}}$ has an equilibrium in pure strategies.

Proof It follows from the equilibrium existence theorem in a generalized game of Debreu (1952) that, in the first place, the objective functions of the agents are continuous and quasi-concave in their strategies. In second place, so are those of the auctioneers, since they are linear, except in the case of those who are choosing the default rates. Finally, the consumer's best response correspondences are convex-valued and upper semicontinuous ${ }^{6}$ on the strategies of fictitious agents;

\footnotetext{
${ }^{6}$ This follows from Hildenbrant (1974, p.26, fact 4), since the initial endowments are strictly positive and the commodity prices belong to the simplex.
} 
and the best response correspondences of the $l+f$ fictitious agents are convexvalued and upper semicontinuous on the consumer's strategies as well. Then we can apply Kakutani's fixed point theorem to best response correspondences to find the equilibrium.

Lemma 3 The equilibrium of the game $\mathcal{G}^{\mathcal{N}}$ is an equilibrium, with a strictly positive price system, for $\mathcal{E}^{\mathcal{N}}$ for a $N$ large enough.

Proof Let $\left(p_{n}^{N}, q_{n}^{N},\left(x_{n}^{h N}\right)_{h \in \cup_{n=-N}^{N} J_{n}}\right)_{-N \leq n \leq N}$ be an equilibrium in pure strategies for $\mathcal{G}_{N}$. We take $n$ so that both young agent $h \in J_{n}$ and his antecessor $f^{-1}(h)$ do not live in the end horizons of the $\mathcal{E}^{N}$. This is obtained if $N$ is large enough. Notice that we lose nothing by analyzing the intermediate agents, since our only interest is in the asymptotic properties.

Then, by definition of equilibrium, we have that

$$
\begin{gathered}
p_{n}^{N}(\tilde{s})\left[c_{o}^{h N}(\tilde{s})-\omega_{o}^{h N}(\tilde{s})\right]+q_{n}^{N}(\tilde{s})\left[y_{o}^{h N}(\tilde{s})-z_{o}^{h N}(\tilde{s})\right] \leq 0 \\
p_{n+1}^{N}\left(\tilde{s}, s^{\prime}\right)\left[c^{h N}\left(\tilde{s}, s^{\prime}\right)-\omega^{h N}\left(\tilde{s}, s^{\prime}\right)\right]+q_{n+1}^{N}\left(\tilde{s}, s^{\prime}\right)\left[y^{h N}\left(\tilde{s}, s^{\prime}\right)-z^{h N}\left(\tilde{s}, s^{\prime}\right)\right] \leq \\
\left(1-k_{n+1}^{N}\left(\tilde{s}, s^{\prime}\right)\right) p_{n+1}^{N}\left(\tilde{s}, s^{\prime}\right) A_{n+1}\left(\tilde{s}_{n}, s^{\prime}\right)\left\{y_{o}^{h N}(\tilde{s})+y^{f^{-1}(h N)}(\tilde{s})\right\} \\
-\left\{D_{n+1}^{h N}\left(\tilde{s}_{n}, s^{\prime}\right)+p_{n+1}\left(\tilde{s}_{n}, s^{\prime}\right) A_{n+1}\left(\tilde{s}_{n}, s^{\prime}\right) z_{o}^{h N}\right\}, \forall s^{\prime} \in S
\end{gathered}
$$

Since the antecessor $f^{-1}(h)$ of $h \in J_{n}$ is old in period $n$, his budget set in that period is then

$$
\begin{gathered}
p_{n}^{N}(\tilde{s})\left[c^{f^{-1}(h N)}(\tilde{s})-\omega^{f^{-1}(h N)}(\tilde{s})\right]+q_{n}^{N}(\tilde{s})\left[y^{f^{-1}(h N)}(\tilde{s})-z^{f^{-1}(h N)}(\tilde{s})\right] \leq \\
\left(1-k_{n}^{N}(\tilde{s})\right) p_{n}^{N}(\tilde{s}) A_{n}(\tilde{s})\left\{y_{o}^{f^{-1}(h N)}(\tilde{s})+y^{f^{-2}(h N)}\left(\tilde{s}^{-}\right)\right\} \\
-\left\{D_{n}^{f^{-1}(h N)}(\tilde{s})+p_{n}^{N}\left(\tilde{s}_{n}\right) A_{n}(\tilde{s}) z_{o}^{f^{-1}(h N)}(\tilde{s})\right\}, \forall s \in S
\end{gathered}
$$

with $\tilde{s}_{n}=\left(\tilde{s}_{n}^{-}, s\right)$. Adding over $h \in J_{n}$ in (A.1) and (A.3) separately and then summing them up we have

$$
p_{n}^{N}\left(\tilde{s}_{n}\right) \sum_{h \in J_{n}}\left[c_{o}^{h N}(\tilde{s})+x^{f^{-1}(h N)}(\tilde{s})-\omega_{o}^{h N}(\tilde{s})-\omega^{f^{-1}(h N)}(\tilde{s})\right]
$$




$$
\begin{gathered}
+q_{n}^{N}(\tilde{s}) \sum_{h \in J_{n}}\left[y_{o}^{h N}(\tilde{s})+y^{f^{-1}(h N)}(\tilde{s})-z_{o}^{h N}(\tilde{s})-z^{f^{-1}(h N)}(\tilde{s})\right] \leq \\
\left(1-k_{n}^{N}(\tilde{s})\right) p_{n}^{N}(\tilde{s}) A_{n}(\tilde{s}) \sum_{h \in J_{n}}\left\{y_{o}^{f^{-1}(h N)}\left(\tilde{s}^{-}\right)+y^{f^{-2}(h N)}\left(\tilde{s}^{-}\right)\right\} \\
\quad-\sum_{h \in J_{n}}\left\{D^{f^{-1}(h N)}(\tilde{s})+p_{n}^{N}(\tilde{s}) A_{n}(\tilde{s}) z_{o}^{f^{-1}(h N)}\left(\tilde{s}^{-}\right)\right\}
\end{gathered}
$$

The optimality conditions of the fictitious agents' problems imply

$$
\begin{gathered}
\left(1-k_{n}^{N}(\tilde{s})\right) p_{n}^{N}(\tilde{s}) A_{n}(\tilde{s}) \sum_{h \in J_{n}}\left\{y_{o}^{f^{-1}(h N)}\left(\tilde{s}^{-}\right)+y^{f^{-2}(h N)}\left(\tilde{s}^{-}\right)\right\} \\
=\sum_{h \in J_{n}}\left\{D^{f^{-1}(h N)}(\tilde{s})+p_{n}^{N}(\tilde{s}) A_{n}(\tilde{s}) z_{o}^{f^{-1}(h N)}\left(\tilde{s}^{-}\right)\right\}
\end{gathered}
$$

That is, the suffered default is equal to the given default.

Replacing (A.5) in (A.4) we have

$$
\begin{gathered}
p_{n}^{N}(\tilde{s}) \sum_{h \in J_{n}}\left[c_{o}^{h N}(\tilde{s})+x^{f^{-1}(h N)}(\tilde{s})-\omega_{o}^{h}(\tilde{s})-\omega^{f^{-1}(h)}(\tilde{s})\right] \\
+q_{n}^{N}(\tilde{s}) \sum_{h \in J_{n}}\left[y_{o}^{h N}(\tilde{s})+y^{f^{-1}(h N)}(\tilde{s})-z_{o}^{h N}(\tilde{s})-z^{f^{-1}(h N)}(\tilde{s})\right] \leq 0
\end{gathered}
$$

The optimality conditions of the auctioneers' problems and (A.6) imply

$$
\begin{gathered}
\sum_{h \in J_{n}}\left[c_{o}^{h N}(\tilde{s})+x^{f^{-1}(h N)}(\tilde{s})-\omega_{o}^{h}(\tilde{s})-\omega^{f^{-1}(h)}(\tilde{s})\right] \leq 0 \\
\sum_{h \in J_{n}}\left[y_{o}^{h N}(\tilde{s})+y^{f^{-1}(h N)}(\tilde{s})-z_{o}^{h N}(\tilde{s})-z^{f^{-1}(h N)}(\tilde{s})\right] \leq 0
\end{gathered}
$$

otherwise the auctioneer could choose other prices for which the value of the aggregate demand is greater.

For a large enough $N$, one has that $p_{n}^{N}$ is a strictly positive vector. Otherwise, every agent $h$ and his antecessor $f^{-1}(h)$ would choose $x_{n}^{h N}=N$ and $x_{n}^{f^{-1}(h N)}=N$, therefore, we will have contradicted (A.7). In a similar way, one has that $q_{n}^{N}>>0$, otherwise, the agent $h$ and his antecessor $f^{-1}(h)$ would choose $y_{n}^{h N}=N$ and $y_{n}^{f^{-1}(h N)}=N$, contradicting (A.8) due to the existence of uniform lower bounds on short sales.

Hence, when $p_{n}^{N}$ and $q_{n}^{N}$ are strictly positive vectors we must have (A.7) and (A.8) with equality implying that all the markets clear in each truncated economy $\mathcal{E}^{N}$. As suffered default is equal to given default by (A.5), Lemma 2 is done. 


\section{Asymptotic Properties of the Sequence of Equilibria}

Let $\left\{\left(x_{n}^{h N}\right)_{-N \leq n \leq N}, p_{n}^{N}, q_{n}^{N}, k_{n}^{N}\right\}_{N \in Z}$ be the sequence of equilibria where each element of the sequence corresponds to an equilibrium of $\mathcal{E}^{N}$ for a large enough $N$. Then, by definition, for any $n$ far away from end horizons, one has

$$
\begin{gathered}
\sum_{h \in J_{n}}\left[c_{o}^{h N}(\tilde{s})+c^{f^{-1}(h N)}(\tilde{s})-\omega_{n}^{h}(\tilde{s})-\omega^{f^{-1}(h)}(\tilde{s})\right]=0 \\
\sum_{h \in J_{n}}\left[y_{o}^{h N}(\tilde{s})+y^{f^{-1}(h N)}(\tilde{s})-z_{o}^{h N}(\tilde{s})-z^{f^{-1}(h N)}(\tilde{s})\right]=0 \\
\left(1-k_{n}^{N}(\tilde{s})\right) p_{n}^{N}(\tilde{s}) A_{n}(\tilde{s}) \sum_{h \in J_{n}}\left\{y_{o}^{f^{-1}(h N)}\left(\tilde{s}^{-}\right)+y^{f^{-2}(h N)}\left(\tilde{s}^{-}\right)\right\} \\
=\sum_{h \in J_{n}}\left\{D^{f^{-1}(h N)}(\tilde{s})+p_{n}^{N}(\tilde{s}) A_{n}(\tilde{s}) z_{o}^{f^{-1}(h N)}\left(\tilde{s}^{-}\right)\right\}
\end{gathered}
$$

and all agents (both $h$ and $f^{-1}(h)$ ) in the period $n$, given the history $\tilde{s}_{n} \in S^{n}$, choose optimality in their truncated budget sets, i.e.,

$$
x_{n}^{h N}\left(\tilde{s}_{n}\right)=\underset{x_{n}^{N} \in B_{n}^{h}\left(\mathbf{p}_{\mathbf{n}}, \mathbf{q}_{\mathbf{n}}, k_{n}\right)}{\operatorname{ArgMax}} U^{h}\left(\mathbf{c}, \mathbf{y}, \mathbf{z}, D, \tilde{s}_{n}\right)
$$

Notice that (A.5) has only been rewritten for completeness.

The sequence $\left\{\left(p_{n}^{N}, q_{n}^{N}, k_{n}^{N}\right)\right\}_{N}$ is bounded, then - passing to a subsequence if it is necessary- converges to $\left(p_{n}, q_{n}, k_{n}\right) \in \triangle^{L-1} \times \triangle^{J-1} \times[0,1]$. On the other hand, (A.9), (A.10) imply that the sequence $\left\{\mathbf{c}_{n}^{h N}(\tilde{s}), \mathbf{y}_{n}^{h N}(\tilde{s}), \mathbf{z}_{n}^{h N}(\tilde{s})\right\}_{N \in Z}$ is bounded and therefore, without loss of generality, we can suppose that it converges, say, to $\left(\mathbf{c}_{n}^{h}(\tilde{s}), \mathbf{y}_{n}^{h}(\tilde{s}), \mathbf{z}_{n}^{h}(\tilde{s})\right)$. Now, from Equation (A.5) it follows that for every $-N \leq$ $n \leq N$, for every $j \in J$, for every $l \in L$, and for every $\tilde{s} \in S^{n}$

$$
D_{n l}^{h j N}(\tilde{s}) \leq 2 v H\left\|A_{n}^{j}(\tilde{s})\right\|
$$

There then exists a subsequence $\psi_{n}: Z \rightarrow Z$ depending on $n$ such that $D_{n l}^{h j \psi_{n}(N)}(\tilde{s})$ converging, say, to $D_{n l}^{h j}$ which is still bounded from above by $2 v H\left\|A_{n}^{j}(\tilde{s})\right\|$.

\section{Proof of Theorem 1}

We will prove that the newly found sequences $\left\{\left(p_{n}, q_{n}, k_{n}\right),\left(x_{n}^{h}\right)\right\}_{n \in Z}$ are an equilibrium for $\mathcal{E}$. First, we demonstrate that all markets clear at prices $\left(p_{n}, q_{n}, k_{n}\right)$, and then $\left(x_{n}^{h}\right)$ are optimal choices in the limit economy.

1. Markets clear and the default rate is correctly anticipated: It follows, from (A.9), (A.10) and (A.5), after taking limit when $N$ goes to infinity; 
2. Optimality: In the first place, notice that $x_{n}^{h N}$ belongs to the truncated budget set. Then the budget feasibility follows after taking limit in truncated budget constraints as $N \rightarrow \infty$.

We will now show that $x_{n}^{h}$ maximizes $U^{h}$ at the cluster point of the sequence $\left\{\left(p_{n}^{N}, q_{n}^{N}, k_{n}^{N}\right)\right\}_{N}$. Suppose that $x_{n}^{h}$ does not maximize $U^{h}$ in the set $B_{n}^{h}\left({ }_{n},{ }_{n}, k_{n}\right.$, $\left.y^{f^{-1}(h)}, z^{f^{-1}(h)}\right)$, then there exists $\bar{x}_{n}^{h} \in B_{n}^{h}\left({ }_{n},{ }_{n}, k_{n}, y^{f^{-1}(h)}, z^{f^{-1}(h)}\right)$ such that $U^{h}\left(\bar{x}_{n}^{h}\right)>U^{h}\left(x_{n}^{h}\right) .{ }^{7}$ By the lower semicontinuity of the budget set, there is $\bar{x}_{n}^{h N} \in$ $B_{n}^{h N}\left(\mathbf{p}_{n}^{N}, \mathbf{q}_{n}^{N}, k_{n}^{N}, y^{f^{-1}(h N)}, z^{f^{-1}(h N)}\right)$ such that $x_{n}^{h N} \rightarrow \bar{x}_{n}^{h}$ as $N \rightarrow \infty$. From the continuity of $U^{h N}$ it follows that

$$
U^{h N}\left(\bar{x}_{n}^{h N}\right)>U^{h N}\left(x_{n}^{h N}\right), \forall N \geq N_{o},
$$

contradicting the optimality of $x_{n}^{h N}$ in the truncated economy $\mathcal{E}^{N}$. Hence, the sequence $\left\{x_{n}^{h}\right\}_{n \in Z}$ is optimal in the limit economy $\mathcal{E}$. This fact implies that nonarbitrage necessary conditions are satisfied in the commodity markets, due to the strictly monotonicity of utility functions, at the cluster point implying $\lim _{N \rightarrow \infty} p_{n}^{N}>>0, \forall n \in Z$.

\footnotetext{
${ }^{7}$ We are dropping the history $\tilde{s} \in S^{n}$ for simplicity. 